\title{
MEAN PLATELET VOLUME IN NEONATAL SEPSIS- A CASE CONTROL STUDY
}

\author{
Mohankumar M. K.1, Salu Paulose ${ }^{2}$
}

1 Professor, Department of Paediatrics, Government Medical College, Thrissur, Kerala, India.

2Junior Resident, Department of Paediatrics, Government Medical College, Thrissur, Kerala, India.

\section{BACKGROUND}

ABSTRACT

The size of platelets in circulation is indicated by Mean Platelet Volume (MPV). It is automatically measured while doing complete blood count in auto analyser. Newborn babies of mothers having risk factors for sepsis are screened for early detection of sepsis. Early signs and symptoms of septicaemia are nonspecific. Hence MPV as an additional parameter of neonatal sepsis will be very much valuable. This study aims to determine the role of Mean Platelet Volume as an additional marker in the diagnosis of neo natal sepsis (NS).

\section{METHODS}

This case-control study was conducted over a period of two years at Neonatal Intensive care Unit (NICU), Institute of Maternal and Child Health, Government Medical College, Calicut, Kerala. A total of 445 newborns were divided into 3 groups: Group A- Culture proven NS ( $\mathrm{n}=39)$, Group B- Clinical NS ( $\mathrm{n}=183)$, and Group C- Apparently healthy control $(\mathrm{n}=223)$. All patients in Group A \& B were subjected to adequate assessment of history, full clinical examination, complete blood count including MPV, C-reactive protein (CRP) and blood culture. In group C, complete blood count including MPV was done and other investigations relevant to the case.

\section{RESULTS}

Septic neonates showed statistically higher values of MPV than control group. The diagnostic cut-off values of MPV for neonatal sepsis was taken as $10 \mathrm{fL}$. The mean MPV in culture proven NS (group A) was 10.9467. In clinical NS (group B) it was 10.4021 and in control (group C) it was 9.3509 .

\section{CONCLUSIONS}

This study shows that MPV has a role as an additional marker in the diagnosis of neonatal sepsis.

\section{KEY WORDS}

Mean Platelet Volume, Neonatal Sepsis, Procedural Sedation, Midazolam, Intranasal, Intravenous

HOW TO CITE THIS ARTICLE: Mohankumar M. K, Paulose S. Mean platelet volume in neonatal sepsis- a case control study. J. Evolution Med. Dent. Sci. 2019;8(22):1808-1812, DOI: 10.14260/jemds/2019/397

\section{BACKGROUND \\ Neonatal sepsis is an important cause of mortality and morbidity in neonates. It is estimated that Neonatal sepsis is responsible for one and a half million deaths each year worldwide.[1] Neonatal sepsis is a clinical syndrome of bacteraemia characterized by systemic signs and symptoms of infection in the first month of life.[2] Neonatal sepsis by itself contributes to $38 \%$ of all deaths in neonates worldwide. Situation is even worse in underdeveloped countries. As per the National Neonatal Perinatal Database 2002-2003 India, the incidence of neonatal sepsis is 30 per 1000 live births. \\ Early onset sepsis is that occurring $<72$ hours of age and the source of infection is mostly maternal genital tract. Late onset sepsis in $>72$ hours and the source of infection is mostly nosocomial, or community acquired.[2] Neonates can deteriorate rapidly, so rapid diagnosis and prompt management is required.}

'Financial or Other Competing Interest': None.

Submission 13-04-2019, Peer Review 16-05-2019,

Acceptance 23-05-2019, Published 03-06-2019.

Corresponding Author:

Dr. Salu Paulose,

PG Hostel,

Government Medical College,

M. G. Kavu, Thrissur-680596,

Kerala, India.

E-mail: salususanpaul@gmail.com

DOI: $10.14260 /$ jemds $/ 2019 / 397$
Sepsis related mortality and morbidity are largely preventable with rational antimicrobial therapy and aggressive supportive care if started earlier.

Newborn babies of mothers having risk factors are screened for early detection of sepsis. The risk factors associated with increased risk for early onset sepsis are Low birth weight $(<2500 \mathrm{gm})$ or prematurity, Febrile illness in mother with evidence of bacterial infection within 2 weeks prior to delivery, Foul smelling and/or meconium stained liquor, Rupture of membranes $>24$ hours, Single unclean or $>$ 3 sterile vaginal examinations during labour, Prolonged labour $>24$ hours (sum of stage 1 and 2), Perinatal asphyxia with Apgar score of $<4$ at 1 minute. Foul smelling liquor alone or presence of two risk factors warrant sepsis screening. Usual sepsis screening methods are the following and considered positive if 2 are present. $\mathrm{TC}<5000 /$ ANC $<1800 /$ IT ratio $>0.2 /$ Micro ESR $>15$ in $1^{\text {st }}$ hour/ CRP +ve $>1 \mathrm{mg} / \mathrm{dL}$. [2] Platelet count and Mean Platelet Volume (MPV) are part of Complete Blood Count (CBC) examination. Thus, evaluating MPV can be an additional marker of neonatal sepsis. MPV measures the average size of platelets in the blood.[3] Statistically significant difference with regard to baseline MPV values between patients with sepsis (Culture proven or Clinical) and healthy controls was found \& MPV can be used in addition to CRP \& IL- 6 in the diagnosis \& follow up of neonatal sepsis.[4]

Sepsis affects almost every organ of the body, including the hematologic system. The clinical features of neonatal 
sepsis are mostly non-specific. A high index of suspicion is needed for early diagnosis. Hypothermia or fever, Lethargy, poor cry, refusal to suck, Poor perfusion, prolonged capillary filling time, Hypotonia, absent neonatal reflexes, Brady/tachycardia, Respiratory distress, apnoea, gasping respiration, Hypo/hyperglycaemia and Metabolic acidosis. Specific features are related to involvement of various systems such as central nervous system, cardiovascular system, gastrointestinal system, hepatic, renal, hematologic and skin. Survivors of neonatal sepsis can have severe neurological sequel due to meningitis as well as hypoxemia resulting from septic shock and severe parenchymal lung disease. The definitive diagnosis of sepsis is only by blood culture. Its positivity rate is low and is affected by blood volume inoculated, prenatal antibiotic use, level of bacteraemia and laboratory capabilities.[5] Moreover there is time delay in obtaining culture reports. Hence the need for other tests for early prediction of sepsis and to follow up the response of antibiotics

Complete Blood Count (CBC) is the most commonly ordered test during the evaluation of neonatal sepsis work up. CBC is done using automated coulter counters. These analysers rapidly measure platelet count and platelet indices. Although important platelet indices are readily available in this CBC result, they are less studied and utilised. The platelet indices related to platelet morphology and kinetics are mean platelet volume (MPV), platelet volume distribution width (PDW) and platelet crit (PCT). Platelet indices are markers of platelet activation.

Among the platelet indices, MPV is the most commonly studied index in neonatal sepsis. MPV is the arithmetic mean of volume of platelets derived from platelet histogram on automated coulter counters. It is expressed in femtoliters (fL). Neonatal sepsis is often associated with decreased platelet production. When platelet production is decreased, young platelets that are bigger and more active enter into circulation and hence the level of MPV increases. Increased MPV indicates increase in platelet diameter. The average MPV in healthy human subjects including adults ranges from 7.2 to $11.7 \mathrm{fL}$. The paucity of gestational age based normative data for MPV has limited the usefulness of MPV. However, it will be more useful to compare baseline MPV and subsequent values while evaluating neonatal sepsis.

\section{Aim of The Study}

The aim of this study is to determine the role of mean platelet volume (MPV) as an additional marker of neonatal sepsis.

\section{METHODS}

\section{Study Design}

Case control study.

\section{Study Setting}

Institute of Maternal and Child Health (IMCH), Medical College, Calicut.

\section{Duration of Study}

From 2015- 2017.

\section{Study Subjects}

All newborn babies with risk factor for sepsis.

\section{Inclusion Criteria}

Hospital delivered babies at IMCH having risk factors for neonatal sepsis.

\section{Exclusion Criteria}

Neonates with congenital anomalies, Hypoxic ischemic encephalopathy, Congenital and acquired causes of thrombocytopenia other than Neonatal sepsis, were excluded.

\section{Justification of Sample Size}

$$
\begin{aligned}
& n=\frac{(Z \alpha+Z \beta)^{2}\left(\sigma_{1}^{2}+\sigma_{2}^{2}\right)}{d^{2}} \\
& \quad n=\frac{(1.96+0.84)^{2}\left(0.64^{2}+1.36^{2}\right)}{0.5^{2}} \\
& \mathrm{n}=70.8=71 \\
& \text { Value of } Z \alpha \text { for } 95 \%-1.96 \\
& \text { Value of } Z \beta \text { for } 80 \%-0.84 \\
& \text { Value of } \mathrm{d}-0.5 \\
& \text { Minimum } 71 \text { cases and controls each. }
\end{aligned}
$$

Based on study conducted by Tiwari, Mahtabuddin, Ahmed and Sharma in, G.C.R.G. Institute of Medical Sciences, Lucknow, Uttar Pradesh, 2017

\section{Method of Sample Collection}

Approval of Institutional Ethical Committee was obtained. Consent was obtained from parents of patients whom were willing to participate in this study.

The neonates participating in the study were classified into three groups-

\section{Group A- Culture Proven NS}

Clinical symptoms of neonatal sepsis and blood culture +ve.

\section{Group B- Clinical NS}

Clinical symptoms of neonatal sepsis, sepsis screen +ve, but blood culture -ve, and need antibiotics for $>7$ days.

\section{Group C- Control}

No clinical symptoms of sepsis.

All newborn babies born in IMCH were evaluated for risk for sepsis. All neonates with 2 risk factors for neonatal sepsis (except foul smelling liquor which by itself is sufficient) were subjected to sepsis screening tests. Those with 1 risk factor was closely observed \& sepsis screening done if they develop any clinical symptoms of neonatal sepsis.

The clinical symptoms of sepsis were noted down as and when it occurred from admission to discharge/death.

These include fever, hypothermia, lethargy, refusal to suck, respiratory distress, apnoea, increased capillary filling time, seizure, necrotizing enterocolitis, mottling, jaundice, a/c renal failure, bleeding, umbilical sepsis. Sepsis screening was done as soon as the newborn fulfils screening criteria or the occurrence of first clinical symptom.

\section{Laboratory Investigations}

CBC in five-part analyser (Sysmex), C- Reactive protein (CRP) and Blood culture (BACTEC method) were done when two 
risk factors of sepsis were present, (Except foul smelling liquor, which alone is sufficient to do the tests). CRP if negative, was repeated at 48 hours of age.

\section{Statistical Analysis}

Statistical analysis was performed using statistical package for social sciences (SPSS Ver.18.0) statistics analysing software. The quantitative data obtained were expressed as mean \pm Standard Deviation and qualitative data were expressed as percentage and frequency.

Receiver operating characteristic (ROC) curve analysis was used to find out the sensitivity and specificity and also to find the best cut-off value for MPV in neonatal sepsis. The two means were compared using Independent student $t$ test.

Full clinical examination was done to assess gestational age, birth weight, detection of clinical signs and symptoms of neonatal sepsis throughout the course of illness until discharge/death.

\section{RESULTS}

Out of 445 cases, 222 were in the patient group, which was again subdivided as group A (culture proven sepsis) - cases, and group B (clinical sepsis)-cases. There were 223 cases in group C (control).

The most frequent symptoms of NS in this study were refusal to suck (43.5\%), lethargy (30.9\%), respiratory distress (21.1\%), hypothermia (14.3\%), and fever (9.4\%) (Table 1).

The results of lab investigations Total WBC count (TC), Absolute Neutrophil Count (ANC), Platelet count (plt)per $\mathrm{mm} 3, \mathrm{C}$-reactive protein (CRP) in $\mathrm{g} / \mathrm{L}$. were as follows. (Table 2)

These results show that difference exist between sepsis group (A-culture proven, B-clinical) against control group (Ccontrol group) which are statistically significant.

The comparison of MPV among the three groups shows a statistically increase in MPV in group A and B (Cut off $10 \mathrm{fl}$ ) than group C. The mean MPV in culture proven NS (group A) was 10.9467, in clinical NS (Group B) it was 10.4021 and in control (group C) it was 9.3509 (Table 3). These results show that a statistically significant difference exists between MPV in sepsis group and control group $(\mathrm{p}<0.001)$

Out of 222 cases in the study group, $39(17.57 \%)$ cases were culture proven sepsis. Of the 39-culture proven sepsis, Staphylococcus aureus 13 (33.33\%), Coagulase negative staphylococcus 9 (23.07\%), Methicillin resistant Staphylococcus aureus 8 (20.51\%), Klebsiella pneumoniae 4 (10.26\%), Candida 3 (7.69\%), Pseudomonas aeruginosa $1(2.56 \%)$ and Acinetobacter 1 (2.56\%) (Table 4)

Receiver Operating Characteristic (ROC) curve shows the relationship between clinical sensitivity and specificity for every possible cut-off for a test or a combination of tests. In addition, the area under the ROC curve gives an idea about the benefit of using the test(s) in question. The ROC curve is a graph with $\mathrm{X}$-axis showing 1 - specificity (= false positive fraction $=\mathrm{FP} /(\mathrm{FP}+\mathrm{TN}))$ and the $\mathrm{Y}$-axis showing sensitivity $(=$ true positive fraction $=\mathrm{TP} /(\mathrm{TP}+\mathrm{FN})$ ).

When ROC curve is used to detect the best cut off point of MPV in neonatal sepsis in this study, it was observed that the area under the curve was 0.871 and the cut-off point for MPV was $10 \mathrm{fl}$, with a sensitivity of $74.3 \%$, specificity of $85.2 \%$, positive predictive value of $83.3 \%$, and negative predictive value of $76.9 \%$.
This study shows that MPV has a statistically significant positive correlation with sepsis in newborn.

\begin{tabular}{|c|c|c|}
\hline Clinical Feature & No. & \% (n=222) \\
\hline Refusal to suck & 97 & 43.69 \\
\hline Lethargy & 69 & 31.08 \\
\hline Respiratory distress & 47 & 21.17 \\
\hline Hypothermia & 32 & 14.41 \\
\hline Fever & 21 & 9.46 \\
\hline Seizure & 14 & 6.30 \\
\hline Umbilical sepsis & 12 & 5.41 \\
\hline Jaundice & 11 & 4.95 \\
\hline Apnoea & 7 & 3.15 \\
\hline Increased CFT & 6 & 2.70 \\
\hline Mottling & 4 & 1.80 \\
\hline Bleeding & 3 & 1.35 \\
\hline NEC & 2 & 0.90 \\
\hline Acute renal failure & 2 & 0.90 \\
\hline \multicolumn{2}{|c|}{ Table 1. Symptoms of Neonatal Sepsis } \\
\hline
\end{tabular}

\begin{tabular}{|c|c|c|c|c|c|}
\hline \multicolumn{6}{|c|}{ Group Statistics } \\
\hline & & $\mathrm{N}$ & Mean & Std. Deviation & $P$ values \\
\hline \multirow{3}{*}{ plt } & A & 39 & 214576.92 & 94509.419 & P1-0.08 \\
\hline & $\mathrm{B}$ & 183 & 243248.63 & 91914.390 & P2-0.001 \\
\hline & $\mathrm{C}$ & 223 & 272592.38 & 61899.460 & P3-0.001 \\
\hline \multirow{3}{*}{$\mathrm{TC}$} & 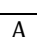 & 39 & 15101.54 & 8172169 & P1-0.927 \\
\hline & $\mathrm{B}$ & 183 & 14913.55 & 12150.280 & P2-0.001 \\
\hline & $\mathrm{C}$ & 223 & 11058.39 & 2376.063 & P3-0.001 \\
\hline \multirow{3}{*}{ ANC } & $\mathrm{A}$ & 39 & 8088.18 & 5928.612 & P1-0.329 \\
\hline & B & 183 & 7237.33 & 4691.973 & P2-0.001 \\
\hline & $\mathrm{C}$ & 223 & 5865.07 & 1785.677 & P3-0.001 \\
\hline & & & & & \\
\hline \multirow{2}{*}{ CRP } & A & 39 & 63.41 & 12.298 & P1-0.001 \\
\hline & $\mathrm{B}$ & 183 & 43.15 & 8.009 & \\
\hline \multicolumn{6}{|c|}{ Table 2. Results of Lab Investigations } \\
\hline \multicolumn{6}{|c|}{$\begin{array}{l}\text { P2- comparison between groups A\&C } \\
\text { P3- comparison between groups } B \& C\end{array}$} \\
\hline
\end{tabular}

\begin{tabular}{|c|c|c|c|c|}
\hline & Mean & N & Std. Deviation & \\
\hline Culture+ & 10.9467 & 39 & .93006 & $\mathrm{P} 1-<0.001$ \\
\hline Clinical & 10.4021 & 183 & .74426 & $\mathrm{P} 2-<0.001$ \\
\hline Control & 9.3509 & 223 & .72224 & $\mathrm{P} 3-<0.001$ \\
Total & 9.9231 & 445 & .95546 & \\
\hline
\end{tabular}

Table 3. Comparison of MPV Among the Three Groups

\begin{tabular}{|c|c|c|}
\hline Organism & Frequency & $\%(n=39)$ \\
\hline Staph aureus & 13 & 33.33 \\
\hline CONS & 9 & 23.07 \\
\hline MRSA & 8 & 20.51 \\
\hline Klebsiella & 4 & 10.26 \\
\hline Candida & 3 & 7.69 \\
\hline Acinetobacter & 1 & 2.56 \\
\hline Pseudomonas & 1 & 2.56 \\
\hline Total & 222 & 100.0 \\
\hline \multicolumn{3}{|c|}{ Table 4. Organisms Causing Neonatal Sepsis } \\
\hline
\end{tabular}

\begin{tabular}{|c|c|}
\hline MPV for Cut Off 10 & \\
\hline Sensitivity & $74.3 \%$ \\
\hline Specificity & $85.2 \%$ \\
\hline Positive predictive value & $83.3 \%$ \\
\hline Negative predictive value & $76.9 \%$ \\
\hline \multicolumn{2}{|c|}{ Validity of MPV in The Diagnosis of Neonatal Sepsis } \\
\hline
\end{tabular}




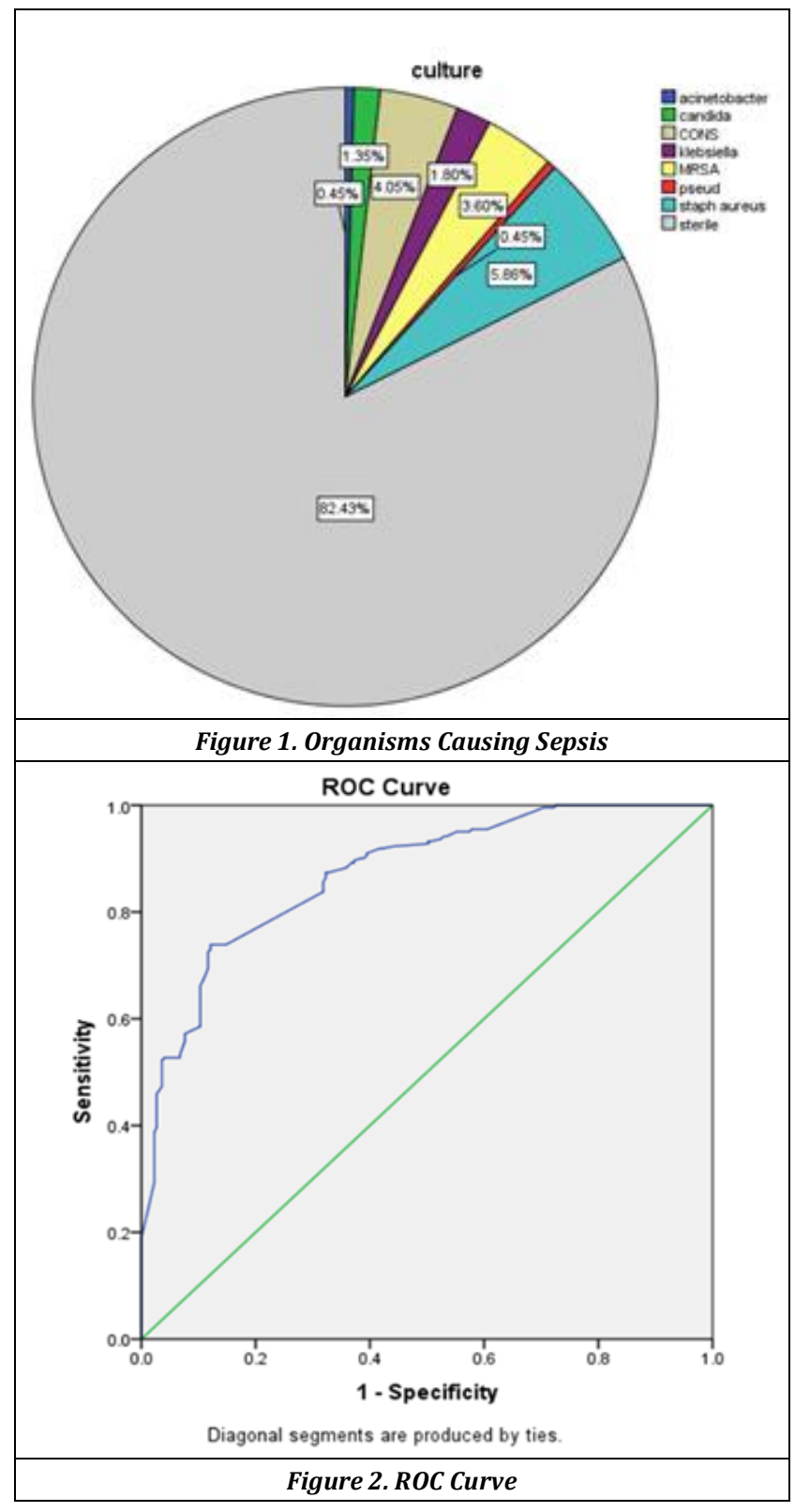

\section{DISCUSSION}

This study shows that the most frequent symptoms of NS were refusal to suck (43.5\%), lethargy (30.9\%), respiratory distress (21.1\%), hypothermia (14.3\%), fever (9.4\%) (Table $2)$. The clinical presentation slightly varied between studies in that the study done by Masoumeh Hematyar et al [6] showed respiratory distress in (44.5\%), jaundice in $(25.5 \%)$, vomiting in $(23.6 \%)$ and poor feeding in $(20.9 \%)$, lethargy $(10.9 \%)$, fever $(3.6 \%)$, seizure $(1.8 \%), \&$ in another study by Mohsen M. Shalaby et al[7] the most common clinical features of neonatal sepsis were poor suckling (87.5\%), respiratory distress (82.5\%) and lethargy (77.5\%).

Among the laboratory investigations for sepsis screening, the total WBC count, Absolute Neutrophil Count (ANC), and Creactive protein (CRP) showed statistically significant increase in group A and B than group C. On comparison of MPV among the three groups, a statistically significant increase in MPV was found in group A and B (cut off $10 \mathrm{fl}$ ) than group $\mathrm{C}(\mathrm{P}<0.001)$. The mean MPV in culture proven NS (group B) was 10.9467, In clinical NS (group A) it was 10.4021 and in control (group C) it was 9.3509. This is similar to the study by Mohsen M Shalaby et al [7] which showed MPV (fl) $10.4 \pm 0.71$ in clinical NS, $10.5 \pm 0.77$ in culture proven NS and $9.46 \pm 0.76$ in control group.

Out of 222 cases in the study group, 39 (17.57\%) cases were culture proven sepsis. Of the 39-culture proven sepsis, Staphylococcus aureus 13(33.33\%), Coagulase negative staphylococcus (CONS) 9 (23.07\%), Methicillin resistant Staphylococcus aureus (MRSA) 8(20.51\%), klebsiella 4 (10.26\%), Candida 3 (7.69\%), Pseudomonas 1(2.56\%) and Acinetobacter 1 (2.56\%). In the study by Mohsen M Shalaby et al,[7] Klebsiella 27.80\%, CONS 16.70\%, MRSA, Pseudomonas, E.coli. Acinetobacter, Candida $11.10 \%$ each. In the study by Suhail Ahmad Naik, Altaf Ahmad et $\mathrm{al},{ }^{[8]}$ Klebsiella $14 \quad(36.8 \%), \quad$ E. coli $12 \quad(31.5 \%)$, Pseudomonas 2 (5.2\%), Acinetobacter 2 (5.2\%), Staph. Aureus 2 (5.2\%), CONS 5 (13.15\%), Candida (2.6\%). This shows that the organisms are more or less the same, but its prevalence is different region wise.

ROC curve in this study for detection of the best cut off point of MPV in neonatal sepsis shows that the area under the curve was 0.871 and the cut-off point for MPV in neonatal sepsis was $10 \mathrm{fl}$, with a sensitivity of $74.3 \%$, specificity of $85.2 \%$, positive predictive value of $83.3 \%$, negative predictive value of $76.9 \%$ and diagnostic accuracy $79.7 \%$. In the study by Mohsen M Shalaby et al,[7] the area under the curve was 0.629 , the cut-off point for MPV was $10.2 \mathrm{fl}$, with a sensitivity of $71 \%$, specificity of $63 \%$, positive predictive value of $74 \%$, negative predictive value of $59 \%$ and diagnostic accuracy of $68 \%$. So $10 \mathrm{fl}$ can be taken as the cut-off point of MPV for neonatal sepsis, above which there is a high probability of neonatal sepsis. Mean platelet volume in children normally ranges from 6 to $10 \mathrm{fL}[9]$.

The role of maternal mean platelet volume levels for the prediction early onset neonatal sepsis in term infants were also studied by Yasemin Cekmez, Merve Dizdar et al,[10] and found that MPV levels were statistically higher in the study group of mothers with infection than the control group. It is easy to predict the probability of sepsis in infants born to mothers who has clinical findings of chorioamnionitis, and other systemic infections. But in mothers who have infection, but not overt to identify, mean platelet volume in mothers can be a predictor of early onset sepsis in newborn. This was not included in this study and further studies may be helpful in this regard.

\section{Limitations}

There may be confounders within the study.

\section{CONCLUSIONS}

MPV increases significantly in neonates with sepsis. So, MPV can be a used as an additional diagnostic marker in neonatal sepsis.

\section{Ethical Approval}

Approved by Institutional Ethical Committee of Medical College, Calicut.

\section{REFERENCES}

[1] Smertka M, Wroblewska J, Suchojad A, et al. Serum and urinary NGAL in septic newborns. Biomed Res Int 2014;2014:717318. 
[2] Sankar MJ, Aggarwal R, Deorari AK, et al. Sepsis in the newborn. Indian Journal of Pediatrics 2008;75(3):2616.

[3] Tiwari R, Mahtabuddin, Ahmed QR, et al. Study of mean platelet volume as predictive index of neonatal sepsis. International Journal of Biomedical Research 2017;8(04):220-3.

[4] Guclu E, Durmaz Y, Karabay O. Effect of severe sepsis on platelet count and their indices. Afr Health Sci 2013;13(2):333-8.

[5] Oncel MY, Ozdemir R, Yurttutan S, et al. Mean platelet volume in neonatal sepsis. J Clin Lab Anal 2012;26(6):493-6.

[6] Hematyar M, Najibpour R. Assessing the role of clinical manifestations and laboratory findings in neonatal sepsis. Archives of Pediatric Infectious Diseases 2017;5(1):e29985.
[7] Shalaby MM, Sobeih AA, Abdulghany WE, et al. Mean platelet volume and serum uric acid in neonatal sepsis: a case-control study. Ann Med Surg (Lond) 2017;20:97-102.

[8] Naik SA, Ahmad A, Irshad M, et al. Clinical profile \& bacteriological spectrum of neonatal sepsis in a tertiary care hospital, Kashmir, India. JEMDS 2019;8(6):346-51.

[9] Orkin SH, Nathan DG, Ginsburg D, et al. Text book of Nathan and Oskis Haematology of infancy and childhood. $7^{\text {th }}$ edn. Philadelphia: Saunders 2008: p. 1380 .

[10] Cekmez Y, Gulecoglu DM, Ozcan C, et al. The utility of maternal mean platelet volume levels for early onset neonatal sepsis prediction of term infants. Ginekologia Polska 2017;88(6):312-4. 